\title{
Mixed form of grave scabies on voluntary cosmetic depigmation land: About a case at the Parakou (Benin) University Hospital Center (UHC)
}

\section{Christiane Koudoukpo', Félix Atadokpèdé ${ }^{2}$, Laouali Salissou ${ }^{3}$, Hugues Adégbidi², Bénédicte Cavelier Balloy4, Fabrice Akpadjan², Nadège Agbéssi ${ }^{1}$, Bérénice Dégboé2, Florence Padonou ${ }^{3}$}

${ }^{1}$ Department of Dermatology and Venerology Unity, Faculty of Medicine of Parakou, University of Parakou, BP 123 Parakou, Benin, ${ }^{2}$ Department of Dermatology and Venerology Unity, Faculty of Health Sciences of Cotonou, University of Abomey Calavi, 01 BP 188 Cotonou, Benin, ${ }^{3}$ Department of Dermatology and Venerology Unity, Faculty of Health Sciences of the Abdou Moumouni University of Niamey, BP 10896 Niamey, Niger, ${ }^{4}$ Department of Anatomical Pathology Unity, Hôpital Saint Louis, 1, Avenue Claude Vellefaux, 75010 Paris, France

Corresponding author: Dr. Laouali Salissou, E-mail: danmata@yahoo.com

\begin{abstract}
Severe forms of scabies are rare. They are more contagious, posing a public health problem during an epidemy in institution. The magnitude of the depigmenting cosmetic practice, a true phenomenon of society today, is at the origin of the recrudescence of certains infectious diseases in their rare forms like severe scaby. We report the first case of mixed form of severe at Parakou University Hospital Center. The body treatment with an antiscabious medicine associated with the disinfection of bedding, led to a total recovering.
\end{abstract}

Key words: Severe scabies; Voluntary cosmetic depigmentation; Dermocorticoid; Benin

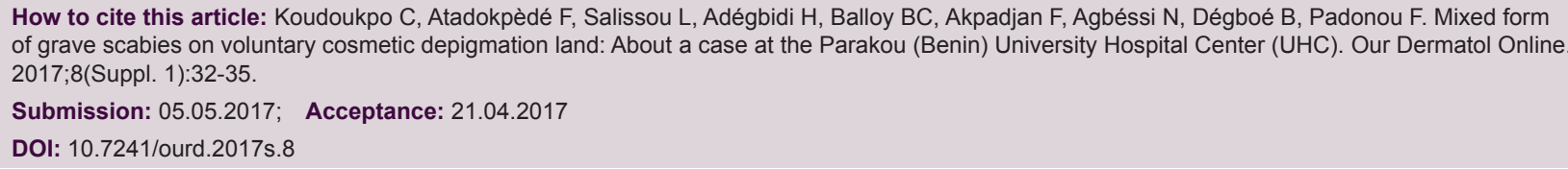




\title{
Forme mixte de gale grave sur terrain de depigmentation cosmetique volontaire: A propos d'un cas au Centre Hospitalier Universitaire (CHU) de Parakou (Benin)
}

\author{
Christiane Koudoukpo', Félix Atadokpèdé2, Laouali Salissou ${ }^{3}$, Hugues Adégbidi², \\ Bénédicte Cavelier Balloy4, Fabrice Akpadjan², Nadège Agbéssi', Bérénice Dégboé2, \\ Florence Padonou ${ }^{3}$
}

\begin{abstract}
${ }^{1}$ Department of Dermatology and Venerology Unity, Faculty of Medicine of Parakou, University of Parakou, BP 123 Parakou, Benin, ${ }^{2}$ Department of Dermatology and Venerology Unity, Faculty of Health Sciences of Cotonou, University of Abomey Calavi, 01 BP 188 Cotonou, Benin, ${ }^{3}$ Department of Dermatology and Venerology Unity, Faculty of Health Sciences of the Abdou Moumouni University of Niamey, BP 10896 Niamey, Niger, ${ }^{4}$ Department of Anatomical Pathology Unity, Hôpital Saint Louis, 1, Avenue Claude Vellefaux, 75010 Paris, France
\end{abstract}

Corresponding author: Dr. Laouali Salissou, E-mail: danmata@yahoo.com

\begin{abstract}
RÉSUMÉ
Les formes graves de la gale sont rares. Elles sont plus contagieuses, posant un problème de santé publique lors d'épidémie en institution. L'ampleur de la pratique cosmétique dépigmentante, véritable phénomène de société de nos jours est à l'origine de la recrudescence de certaines pathologies infectieuses dont des formes rares de gale grave. Nous rapportons le premier cas de forme mixte de gale grave sur terrain de dépigmentation cosmétique volontaire chez une jeune femme de 21 ans au CHU de Parakou. Le traitement corporel par un antiscabieux associé à la désinfection de la literie, a conduit à une guérison totale.
\end{abstract}

Mots-clés: Gale grave; Dépigmentation cosmétique volontaire; Dermocorticoïde; Bénin

\section{INTRODUCTION}

Les formes graves de la gale sont rares. Elles sont plus contagieuses, posant un problème de santé publique lors d'épidémie en institution [1-3]. Il existe deux formes de gale grave: la gale hyperkératosique, anciennement dénommée gale norvégienne, se caractérisant par une hyperkératose pouvant s'étendre sur toute la surface corporelle, dont le visage et le cuir chevelu ou parfois, pouvant aussi être localisée avec un prurit discret voire absent; et la gale disséminée inflammatoire, qui est souvent la conséquence d'un diagnostic tardif, voire de traitements itératifs d'une gale commune avec des corticoïdes locaux [1-3]. Des cas de gale grave sur terrain dépigmentation cosmétique volontaire ont été décrits [4-6]. Nous rapportons cette observation de forme mixte de gale grave sur un terrain de dépigmentation par usage de produits cosmétiques contenant un dermocorticoïde de classe une, dans le but de mettre l'accent sur ce premier cas rare.

\section{OBSERVATION}

Il s'est agit d'une femme de 21 ans, élève aide-soignante, aux antécédents d'usage de produits cosmétiques contenant le Clobétasol proprionate depuis un an. Elle a consulté au CHU de Parakou en janvier 2017 pour des lésions papulo-croûteuses, prurigineuses évoluant depuis octobre 2016, ayant débuté aux fesses, avec une extension profuse. Aucune notion de contage familial n'a été relevée,

\footnotetext{
How to cite this article: Koudoukpo C, Atadokpèdé F, Salissou L, Adégbidi H, Balloy BC, Akpadjan F, Agbéssi N, Dégboé B, Padonou F. Forme mixte de gale grave sur terrain de depigmentation cosmetique volontaire: A propos d'un cas au Centre Hospitalier Universitaire (CHU) de Parakou (Benin). Our Dermatol Online. 2017;8(Suppl. 1):32-35.

Submission: 05.05.2017; Acceptance: 21.07.2017

DOI: 10.7241 /ourd.2017s.8
} 
mais elle a signalé une exacerbation nocturne du prurit. Al'examen clinique, les lésions étaient hyperkératosiques, hyperpigmentées et diffuses (Fig. 1). Les stries croûteuses de grattage siégeaient sur le tronc, les fesses et les membres (Fig. 2). Une dyschromie était localisée au dos des mains. Lexamen histopathologique avait montré la présence d'un Sarcoptes scabiei variété homonis intracorné, entouré de polynucléaires neutrophiles avec un discret infiltrat inflammatoire dermique superficiel à prédominance lymphocytaire (Fig. 3). La sérologie rétrovirale était négative. Le diagnostic de forme mixte de gale grave sur terrain de dépigmentation cosmétique volontaire avait été posé devant l'existence des signes d'hyperkératose diffuse caractérisant la gale norvégienne et la profusion des lésions très prurigineuses. Un traitement corporel fait de Pyréthrinoïde associé à une désinfection de la literie et de la lingerie avec arrêt d'utilisation du produit cosmétique dépigmentant, avait abouti à une guérison totale (Figs. 4a et $4 \mathrm{~b})$ au bout de deux semaines.

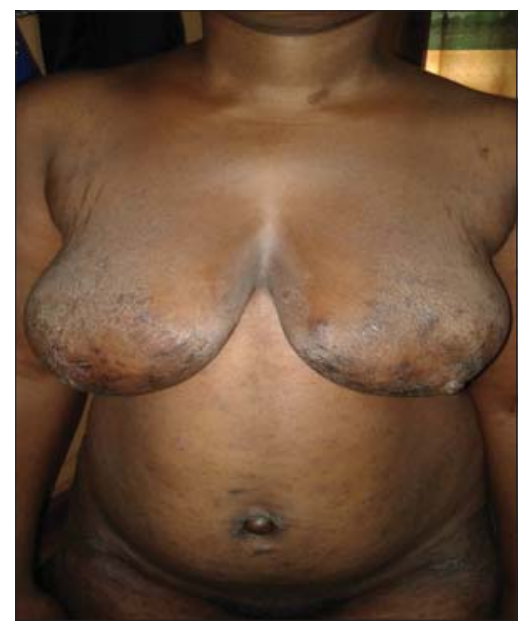

Figure 1: Lésions hyperkératosiques hyperpigmentées diffuses.

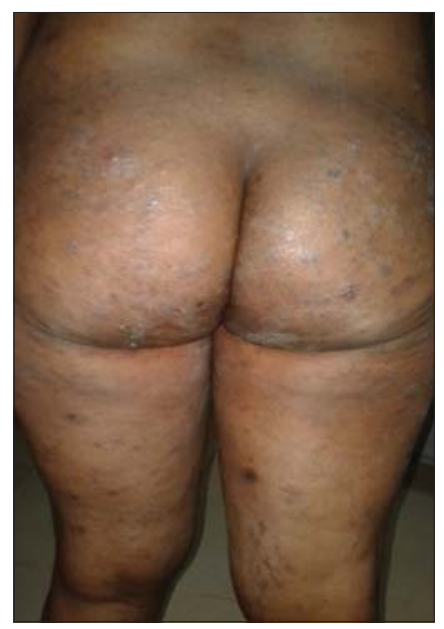

Figure 2: Lésions croûteuses et érosives de grattage.

\section{DISCUSSION}

Le cas clinique exposé présente une particularité morphologique de la gale liée à la pratique cosmétique dépigmentante. En effet si le phénomène de dépigmentation de la peau est bien connu, surtout en Afrique noire et en Amérique, la population reste peu sensible à ses complications qu'on ne cesse néanmoins de décrier [7]. La dépigmentation cosmétique volontaire constitue un problème de santé publique $[8,9]$. Elle consiste en un éclaircissement de la peau par l'utilisation de produits parmi lesquels les dermocorticoïdes d'activité très forte, l'hydroquinone, les produits mercuriels et les décapants comme la soude [10-12]. De l'avis de certains auteurs, la grande fréquence de la gale sur terrain de dépigmentation cosmétique volontaire pourrait s'expliquer par une utilisation prolongée de produits cosmétiques dépigmentants contenant

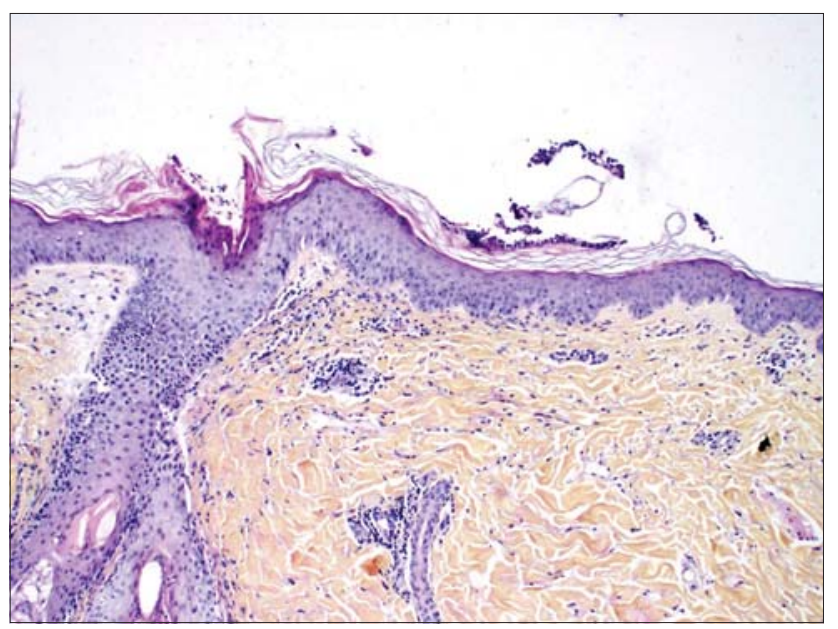

Figure 3: Image d'un Sarcoptes scabiei intracorné, entouré de polynucléaires neutrophiles. avec un discret infiltrat inflammatoire dermique superficiel surtout lymphocytaire.

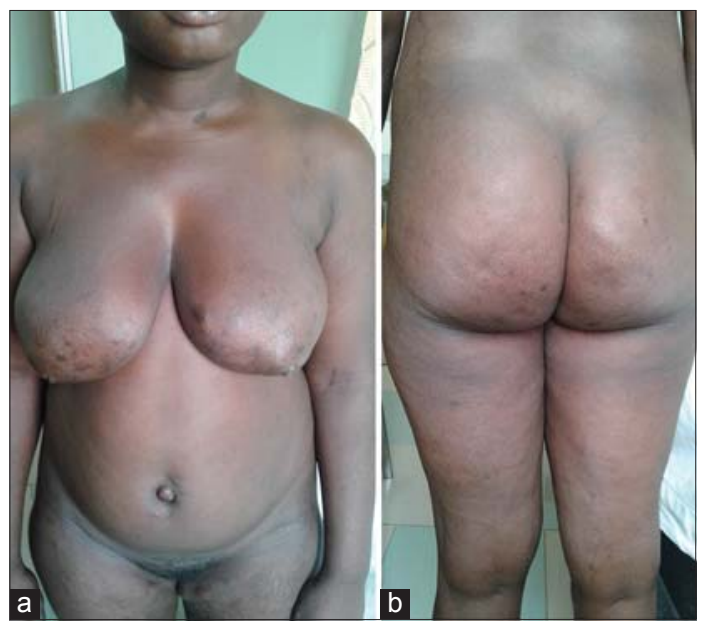

Figure 4: $(a$ et b) Vues antérieure et postérieure après guérison. 
des dermocorticoïdes [4-6]. Il a été démontré que ces dermocorticoïdes détruisent la flore microbienne protectrice, exposant comme dans le cas décrit, les sujets à des complications cutanées infectieuses et à la profusion des lésions $[13,14]$. Cette destruction de la flore microbienne expose le sujet à développer des affections cutanées surtout infectieuses au simple contact avec l'agent pathogène [15]. Il s'ensuit que la source probable de contamination de notre patiente serait le simple contact avec les patients galeux pendant ses stages hospitaliers, vu l'absence de notion de contage familial signalée. Lutilisation du Clobétasol proprionate qui est un dermocorticoïde à niveau d'activité très puissante comme produit cosmétique par notre patiente entraîne une immunodépression cutanée expliquant la profusion des lésions. En raison de l'absence relativement fréquente du prurit chez le sujet âgé et l'immunodéprimé, terrains particuliers des formes graves de gale, la maladie passe longtemps inaperçue chez ces sujets. Les parasites et les lésions prolifèrent rapidement et la peau se recouvre de formations croûteuses, squameuses, parfois épaisses, blanc-jaune souffre $[5,16,17]$. Cette forme croûteuse ou hyperkératosique, jadis appelée "gale norvégienne" très contagieuse, observée sur ces terrains particuliers est responsable d'épidémies dans les centres de gériatrie et les services d'hospitalisation long séjour [18-20]. Chez notre patiente, il ne s'agissait pas de terrain particulier des formes graves de gale, mais l'hyperkératose serait favorisée par l'évolution chronique des lésions. Le prurit discret, voire absent dans cette forme hyperkératosique [18-20] est présent et très intense chez notre patiente. Ceci serait dû à la pratique cosmétique dépigmentante par le Clobétasol propionate, source de l'immunodépression cutanée et par conséquent la profusion des lésions. C'est ce qui explique la dénomination de forme mixte de gale grave, premier cas rapporté.

\section{CONCLUSION}

La forme mixte de gale grave est rare. Elle mérite d'être évoquée en cas de lésions hyperkératosiques diffuses associées à du prurit. C'est le premier cas rapporté en relation avec l'utilisation de cosmétiques contenant du Clobétasol proprionate, dont l'évolution a été favorable par combinaison de traitement antiscabieux et l'arrêt du dermocorticoïde.

\section{RÉFÉRENCES}

1. Bouvresse $\mathrm{S}$, Chosidow O. Ectoparasitose cutanée gale et pédiculose. Rev Prat. 2011;61:867-73.

2. Chosidow O. Clinical practices. Scabies. N Engl J Med. 2006;354:1718-27.

3. Heukelbach J, Feldmeier H. Scabies. Lancet. 2006;367:1767-74.

4. Nathan Rozen J. Cosmetic agents causing endocrinopathy in an African immigrant Can Fam Physician. 2012;58:169-71.

5. Agence française de sécurité sanitaire des produits de santé (AFSSAPS). Evaluation des risques liés à la dépigmentation volontaire. Rapport d'expertise Octobre 2011, 3.

6. Ly F. Complications dermatologiques de la dépigmentation artificielle en Afrique. Ann Dermatol Venereol. 2006;133:899-906.

7. Agbessi O, Selinger R, Khales A, Nassih M, El Khatib K. Quand les complications de la dépigmentation conduisent à l'indication d'une lipectomie abdominale atypique. Pan Afr Med Jr. 2015;22:194.

8. Morand JJ, Ly F, Lightburn E, Mahé A. Complications de la dépigmentation cosmétique en Afrique. Med Trop. 2007;67:627-34.

9. Mahé A. Utilisation cosmétique de produits dépigmentants. Im Dermatol. 2010;1:25-9.

10. Groupe Thématique "peau noire " de la Société Française de Dermatologie. Liste de spécialités utilisées dans un but cosmétique et ayant été signalées comme contenant des substances éclaircissantes médicalement dangereuses. Ann Dermatol Venerol. 2011;138:443-6.

11. Levang J, Eygonnet F, Humbert P. La dépigmentation volontaire à Mayotte ou le "Pandalao". Ann Dermatol Venerol. 2009;136:681-6.

12. Alghamdi A. The use of topical bleaching agents among women: a cross-sectional study of knowledge, attitude and practices. J Eur Acad Dermatol Venerol. 2010;24:1214-9.

13. Lebrun-Vignes B, Chosidow O. Les dermocorticoïdes. Ann Dermatol Venereol. 2004;131:39-48.

14. Lebrun-Vignes B, Bourgault-Villada I, Chosidow O. Corticothérapie locale. Encycl Méd Chir Thérap. 2014;98-900-A-10, p9.

15. Beneton N, Saiag P. Corticostéroïdes topiques cutanés. Rev Prat. 1999;2251-6.

16. Association Française des Enseignants de Parasitologie et Mycologie (ANOFEL). Gale ou scabiose. 8p.

17. Kebe M, Yahya S, Lo B, Ball M. Etude des complications de la dépigmentation artificielle à Nouakchott, Mauritanie, Mali Médical. 2015;30:38-42.

18. Bitar D, Castor C, Che D, Fischer A, Haeghebaert S, Thiolet J-M. La gale est-elle en augmentation en France? Etat des lieux à partir de diverses enquêtes régionales et nationales. 2008-2010. Saint Maurice: InVS; 2011: 23 p.

19. Bitar D, Thiolet JM, Haeghebaert S, Castor C, Poujol I, Coignard B, Che D. La gale en France entre 1999 et 2010: augmentation de l'incidence et implications en santé publique. Ann Dermatol Veneerol. 2012;139:428-34.

20. Gehanno JF - Gestion des épidémies de gale dans les collectivités. Concours Méd. 2013;135:477-80.

Copyright by Christiane Koudoukpo, et al. This is an open-access article distributed under the terms of the Creative Commons Attribution License, which permits unrestricted use, distribution, and reproduction in any medium, provided the original author and source are credited.

Source of Support: Nil, Conflict of Interest: None declared. 